\title{
Anti-Tuberculosis Drug Induced Liver Injury and Ursodeoxycholic Acid
}

\author{
Susanne M. Lang1 ${ }^{*}{ }^{\mathbb{D}}$, Emad Al-Nemnem $^{2}{ }^{\mathbb{D}}$, Helmut Schiffl $^{3}$ \\ ${ }^{1}$ Klinik für Innere Medizin II, Universitätsklinikum Jena, Jena, Germany \\ ${ }^{2}$ Department of Pulmonary \& Critical Care Medicine, Clemenceau Medical Center DHCC, Dubai, United Arab Emirates \\ ${ }^{3}$ Department of Internal Medicine IV, University Hospital Munich, Munich, Germany \\ Email: *susanne.lang@med.uni-jena.de
}

How to cite this paper: Lang, S.M., Al-Nemnem, E. and Schiffl, H. (2020) Anti-Tuberculosis Drug Induced Liver Injury and Ursodeoxycholic Acid. Journal of Tuberculosis Research, 8, 66-72. https://doi.org/10.4236/jtr.2020.82007

Received: May 18, 2020

Accepted: June 26, 2020

Published: June 29, 2020

Copyright (c) 2020 by author(s) and Scientific Research Publishing Inc. This work is licensed under the Creative Commons Attribution International License (CC BY 4.0).

http://creativecommons.org/licenses/by/4.0/

\section{(c) (i) Open Access}

\begin{abstract}
Hepatotoxicity induced by standard anti-tuberculosis drugs (isoniazid, rifampicin, pyrazinamide) can result in significant morbidity and, rarely, even mortality. This major adverse side-effect of anti-tuberculosis treatment has a negative impact on patient adherence and patient outcomes as well as on tuberculosis control. Early recognition and prompt withdrawal of the offending drugs are the most critical interventions in the management of anti-tuberculosis drug-induced liver injury. No drug or herbal extract has been shown until recently to prevent or reverse anti-tuberculosis drug-induced hepatotoxicity. Ursodeoxycholic acid is the only FDA approved drug for the treatment of primary biliary cholangitis and has also been successfully used in various cholestatic liver diseases. Although still experimental, recent controlled clinical studies suggested that oral administration of ursodeoxycholic acid may prevent the onset of anti-tuberculosis drug-induced liver injury and accelerate the recovery of liver injury. These clinical data are supported by experimental models of anti-tuberculosis drug-induced hepatotoxicity. There is an urgent need for further randomized clinical trials to document the promising hepatoprotective properties of ursodeoxycholic acid.
\end{abstract}

\section{Keywords}

Ursodeoxycholic Acid, Hepatotoxicity, Rifampicin, Isoniazid, Pyrazinamide, Tuberculosis

\section{Introduction}

Hepatotoxicity is a frequent and potentially serious adverse effect of standard first line anti-tuberculosis drug (ATD) regimens containing the hepatotoxic compounds isoniazid, rifampicin, and pyrazinamide. There is a wide variety in the 
reported incidence of ATD-induced liver injury in different studies (2\% to $28 \%$ ). This may be attributable to patient characteristics, the drug regimen involved, or the threshold of liver function tests used to define hepatoxicity. The most widely used definitions of ATD-induced liver injury rely on serum alanine aminotransferase activities (ALT) (more than 5 times the upper limit of normal (ULN) and bilirubin concentrations (more than 2 times ULN without symptoms) or ALT levels more than 3 times ULN with clinical symptoms of hepatotoxicity [1] [2] [3].

The clinical presentations of ATD-induced liver injury vary from asymptomatic rises in serum transaminases, to acute hepatitis or even fatal liver failure. The high morbidity and mortality of ATD-induced severe liver injury may require interruption or modification of anti-tuberculosis treatment and reduce the efficacy of tuberculosis therapy. Discontinuation of first line drugs or nonadherence to treatment regimens may cause treatment failure, relapse and drug-resistance which can significantly reduce TB control [4] [5].

The pathogenesis underlying ATD-induced liver injury is poorly understood. Direct toxicity of the primary compound or its metabolites is the main cause but an immunologically mediated response may also play a role [6]. Once hepatotoxicity is diagnosed, all ATDs are temporarily withdrawn and sequential re-challenge of the involved drugs is used to identify the drug causing hepatotoxicity [7]. This can be a time-consuming effort for both patients and caregivers. No hepatoprotective agent has been proved beyond any doubt to be effective in ameliorating ATD-induced hepatotoxicity [8] [9] [10]. New therapies are needed to reduce the incidence and severity of ATD-induced hepatotoxicity.

Ursodeoxycholic acid (UDCA) has been successfully used for the treatment of primary biliary cholangitis and pregnant intrahepatic cholestasis [11]. Since UDCA has a favorable safety profile it seemed reasonable to treat cholestatic drug-induced liver injury (DILI) in a variety of diseases [12]. The usefulness of UDCA as a novel hepatoprotective agent for drug-induced hepatotoxicity was largely demonstrated by case reports and observational studies [13]-[21].

This narrative review describes the available experience with this drug in TB patients receiving standard first line treatment.

\section{UDCA and ATD-Induced Liver Injury}

UDCA is the major bile acid of the bile of black bears and has been used for centuries in traditional Chinese medicine as a remedy for cholestatic liver disease [22]. The therapeutic effects of UDCA for ATD-induced hepatotoxicity have been recognized but the data reported (case reports, case series,) are scarce and inconsistent [23] [24] [25].

We carried out a prospective pilot study to evaluate the hepatoprotective effectiveness of oral UDCA (250 - $500 \mathrm{mg}$, TID) administered to 27 adult patients with TB or non-mycobacterial infections and drug-induced liver injury (ALT levels $>5-6$ ULN or bilirubin levels $>2-3$ ULN) [26]. Despite continuation of standard first line anti-TB treatment, elevated enzymes ALT, AST, ALP and bi- 
lirubin normalized in $78 \%$ of the patients and $19 \%$ of the patients showed a significant reduction in liver tests when UDCA was introduced. There was no significant change in liver enzymes/bilirubin in one patient. Due to the hepatoprotective effects there was no need to reduce drug dose in all patients. They all had radiological and clinical improvement, independent from age, sex, ethnicity, and severity of ATD-induced liver injury. In this series, there was no patient with fulminant hepatic failure. We concluded that oral administered UDCA to TB patients may reverse hepatoxicity of first line anti-tuberculosis drugs.

Borzakova and Reyzis conducted a prospective randomized clinical trial to compare the effects of UDCA versus herbal extracts on the reversal of ATD-induced hepatotoxicity in Russia [27]. The study population included 77 young patients (children, adolescents) with pulmonary TB receiving standard anti-tuberculosis regimens. DILI was serious and ATD had to be stopped prior to randomisation. In group 139 patients were randomized to oral UDCA $(20 \mathrm{mg} / \mathrm{kg}$ once, then 12 - $15 \mathrm{mg}$ per day in 2 - 3 intakes). In group 238 children received herbal extracts (silymarin analogues or Hofetil) for ATD-induced hepatotoxicity. The results showed a more pronounced decrease in liver tests (aminotransferases, bilirubin) in the UDCA group compared to the group of patients receiving herbal extracts. The time to normalization of the liver tests was almost half in the UDCA group compared to the control group (29.6 days vs 49.6 days, $\mathrm{p}>0.05$ ). In patients continuing anti-TB therapy the increase in cholestatic parameters was significantly less in UDCA group than in the controls $(14.7 \%$ vs $50.0 \%, \mathrm{p}<0.05)$.

Berestova et al. conducted two prospective randomized clinical trials to investigate prevention or reversal of ATD-induced hepatoxicity in adult patients with pulmonary TB receiving the standard first line regimen. The patients were randomised to UDCA alone (250 mg twice per day), UDCA in combination with taurine, or to milk thistle extract.

Prophylactic administration of combined UDCA and taurine prevented the onset of anti-TB-induced DILI in most patients. Aminotransferase levels remained within the normal range in most patients, only $20 \%$ of the patients developed moderate and sometimes severe increases of serum liver transaminases. In contrast, most patients receiving prophylactic coadministration of milk thistle extract developed ATD-induced DILI and needed a modification of TB therapy (personal communication).

In a second randomized trial the authors examined the addition of UDCA alone or in combination with taurine to ongoing first-line TB treatment on anti-TB-DILI. The combination of UDCA and taurine resulted in normalisation of transaminases in most of these patients. UDCA alone was associated with a significant reduction in transaminases without complete normalization (Berestova, personal communication).

Carefully designed experimental investigations corroborate the findings of these clinical studies. Chen and co-workers examined the protective effects of UDCA on isoniazid plus rifampicin induced liver injury in mice [28]. The animals were 
orally administered UDCA 30 minutes before isoniazid and rifampicin. UDCA pre-treatment significantly alleviated hepatic apoptosis. The UDCA-mediated protective effects seemed to be associated with its antioxidative and anti-apoptotic effects.

UDCA was well tolerated. None of these prospective clinical studies reported any relevant adverse effects of mid-to long-term UDCA therapy in humans.

\section{Mechanisms of Action of UDCA}

The specific mechanisms underlying the protective effect of UDCA on anti-TB-DILI are unknown.

UDCA acts on the liver through a variety of complex and complementary mechanisms. The main effects are: 1) protection of hepatocytes against bile-acid induced apoptosis 2) protection of cholangiocytes against cytotoxicity of hydrophobic bile acids 3) stimulation of hepatobiliary secretion of bile acids, 4) suppression of immune responses, and 5) anti-inflammatory effects [12] [28].

\section{Perspective of UDCA in TB treatment}

UDCA is an inexpensive and well tolerated medication that has shown the potential to alleviate DILI from standard TB regimens in a small number of prospective studies. In most TB patients raised liver functions tests entail a stop and rechallenge strategy which can be a burden for patients and their treating physicians. Prevention of hepatoxicity by UDCA facilitates patient management and may result in better adherence, especially in an out-patient setting.

\section{Conclusion}

UDCA is a promising therapeutic option for the prevention and treatment of DILI. Further clinical studies are required to validate these results.

\section{Authors' Contributions}

All authors have made substantial contributions to: 1) the analysis and interpretation of data; 2) drafting the article or revising it critically for intellectual content; and 3) all authors approved the submitted version to be published.

\section{Conflicts of Interest}

The authors declare no conflicts of interest regarding the publication of this paper.

\section{References}

[1] Nagarajan, S. and Whitaker, P. (2018) Management of Adverse Reactions to First-Line Tuberculosis Antibiotics. Current Opinion in Allergy and Clinical Immunology, 18, 333-341. https://doi.org/10.1097/ACI.0000000000000462

[2] Saukkonen, J.J., Cohn, D.L., Jasmer, R.M., Schenker, S., Jereb, J.A., Nolan, C.M., Peloquin, C.A., Gordin, F.M., Nunes, D., Strader, D.B., Bernardo, J., Venkataramanan, R. and Sterling, T.R. (2006) An Official ATS Statement: Hepatotoxicity of Antituberculosis Therapy. American Journal of Respiratory and Critical Care Medicine, 174, 935-952. https://doi.org/10.1164/rccm.200510-1666ST 
[3] Tostmann, A., Boeree, M.J., Aarnoutse, R.E., de Lange, W.C., van der Ven, A.J. and Dekhuijzen, R. (2008) Antituberculosis Drug-Induced Hepatotoxicity: Concise Up-to-Date Review. Journal of Gastroenterology and Hepatology, 23, 192-202. https://doi.org/10.1111/j.1440-1746.2007.05207.x

[4] Shang, P., Xia, Y., Liu, F., Wang, X., Yuan, Y., Hu, D., Tu, D., Chen, Y., Deng, P., Cheng, S., Zhou, L., Ma, Y., Zhu, L., Gao, W., Wang, H., Chen, D., Yang, L., He, P., Wu, S., Tang, S., Lv, X., Shu, Z., Zhang, Y., Yang, Z., Chen, Y., Li, N., Sun, F., Li, X., He, Y., Garner, P. and Zhan, S. (2011) Incidence, Clinical Features and Impact on Anti-Tuberculosis Treatment of Anti-Tuberculosis Drug Induced Liver Injury (ATLI) in China. PLoS ONE, 6, e21836. https://doi.org/10.1371/journal.pone.0021836

[5] Song, J.H., Yoon, S.Y., Park, T.Y., Heo, E.Y., Kim, D.K., Chung, H.S. and Lee, J.K. (2019) The Clinical Impact of Drug-Induced Hepatotoxicity on Anti-Tuberculosis Therapy: A Case Control Study. Respiratory Research, 20, 283. https://doi.org/10.1186/s12931-019-1256-y

[6] Ramappa, V. and Aithal, G.P. (2013) Hepatotoxicity Related to Anti-Tuberculosis Drugs: Mechanisms and Management. Journal of Clinical and Experimental Hepatology, 3, 37-49. https://doi.org/10.1016/j.jceh.2012.12.001

[7] (2019) EASL Clinical Practice Guidelines: Drug-Induced Liver Injury. Journal of Hepatology, 70, 1222-1261. https://doi.org/10.1016/j.jhep.2019.02.014

[8] Marjani, M., Fahim, F., Sadr, M., Kazempour Dizaji, M., Moniri, A., Khabiri, S., Tabarsi, P. and Velayati, A.A. (2019) Evaluation of Silymarin for Management of Anti-Tuberculosis Drug Induced Liver Injury: A Randomized Clinical Trial. Gastroenterology and Hepatology from Bed to Bench, 12, 138-142.

[9] Tao, L., Qu, X., Zhang, Y., Song, Y. and Zhang, S.X. (2019) Prophylactic Therapy of Silymarin (Milk Thistle) on Antituberculosis Drug-Induced Liver Injury: A Meta-Analysis of Randomized Controlled Trials. Can Journal of Gastroenterology and Hepatology, 2019, Article ID: 3192351. https://doi.org/10.1155/2019/3192351

[10] Xu, L., Zhang, F., Xu, C., Liu, K.G., Wu, W. and Tian, Y.X. (2017) Is the Prophylactic Use of Hepatoprotectants Necessary in Anti-Tuberculosis Treatment? Chemotherapy, 62, 269-278. https://doi.org/10.1159/000465515

[11] Chascsa, D.M.H. and Lindor, K.D. (2020) Emerging Therapies for PBC. Journal of Gastroenterology, 55, 261-272. https://doi.org/10.1007/s00535-020-01664-0

[12] Simental-Mendia, M., Sanchez-Garcia, A. and Simental-Mendia, L.E. (2020) Effect of Ursodeoxycholic Acid on Liver Markers: A Systematic Review and Meta-Analysis of Randomized Placebo-Controlled Clinical Trials. British Journal of Clinical Pharmacology. https://doi.org/10.1111/bcp.14311

[13] Hassan, A. and Fontana, R.J. (2019) The Diagnosis and Management of Idiosyncratic Drug-Induced Liver Injury. Liver International, 39, 31-41.

https://doi.org/10.1111/liv.13931

[14] Arai, T., Kogi, K., Honda, Y., Suzuki, T., Kawai, K., Okamoto, M., Fujioka, T. and Murata, N. (2018) Lorazepam as a Cause of Drug-Induced Liver Injury. Case Reports in Gastroenterology, 12, 546-550. https://doi.org/10.1159/000492209

[15] Asgarshirazi, M., Shariat, M., Dalili, H. and Keihanidoost, Z. (2015) Ursodeoxycholic Acid Can Improve Liver Transaminase Quantities in Children with Anticonvulsant Drugs Hepatotoxicity: A Pilot Study. Acta Medica Iranica, 53, 351-355. http://acta.tums.ac.ir/index.php/acta/article/view/4878

[16] Bordbar, M., Shakibazad, N., Fattahi, M., Haghpanah, S. and Honar, N. (2018) Effect of Ursodeoxycholic Acid and Vitamin E in the Prevention of Liver Injury from 
Methotrexate in Pediatric Leukemia. Turk Journal of Gastroenterology, 29, 203-209. https://doi.org/10.5152/tjg.2018.17521

[17] Fernandes, C.T., Iqbal, U., Tighe, S.P. and Ahmed, A. (2019) Kratom-Induced Cholestatic Liver Injury and Its Conservative Management. Journal of Investigative Medicine High Impact Case Reports, 7, 1-4. https://doi.org/10.1177/2324709619836138

[18] Ito, T., Ozaki, Y., Son, Y., Nishizawa, T., Amuro, H., Tanaka, A., Tamaki, T. and Nomura, S. (2014) Combined Use of Ursodeoxycholic Acid and Bosentan Prevents Liver Toxicity Caused by Endothelin Receptor Antagonist Bosentan Monotherapy: Two Case Reports. Journal of Medical Case Reports, 8, 250. https://doi.org/10.1186/1752-1947-8-250

[19] Kurokawa, K., Hara, M., Iwakami, S.I., Genda, T., Iwakami, N., Miyashita, Y., Fujioka, M., Sasaki, S. and Takahashi, K. (2019) Cholestatic Liver Injury Induced by Pembrolizumab in a Patient with Lung Adenocarcinoma. Internal Medicine, 58, 3283-3287. https://doi.org/10.2169/internalmedicine.2591-18

[20] Sawada, K., Shonaka, T., Nishikawa, Y., Hasegawa, K., Hayashi, H., Hasebe, T., Nakajima, S., Ikuta, K., Fujiya, M., Furukawa, H. and Okumura, T. (2019) Successful Treatment of Nivolumab-Related Cholangitis with Prednisolone: A Case Report and Review of the Literature. Internal Medicine, 58, 1747-1752.

https://doi.org/10.2169/internalmedicine.2330-18

[21] Wree, A., Dechene, A., Herzer, K., Hilgard, P., Syn, W.K., Gerken, G. and Canbay, A. (2011) Steroid and Ursodesoxycholic Acid Combination Therapy in Severe Drug-Induced Liver Injury. Digestion, 84, 54-59. https://doi.org/10.1159/000322298

[22] Wang, D.Q. and Carey, M.C. (2014) Therapeutic Uses of Animal Biles in Traditional Chinese Medicine: An Ethnopharmacological, Biophysical Chemical and Medicinal Review. World Journal of Gastroenterology, 20, 9952-9975. https://doi.org/10.3748/wjg.v20.i29.9952

[23] Alpana, M., Daga, M.K., Aggarwal, S. and Nidhi, A. (2015) Treatment for Tuberculosis in a Patient with Dubin-Johnson Syndrome. BMJ Case Reports, 2015, pii: bcr2015211123. https://doi.org/10.1136/bcr-2015-211123

[24] Kataoka, K., Kono, Y., Sugimoto, M., Furuichi, Y., Shichiri, M. and Tanaka, Y. (2010) Hepatocyte-Protective and Anti-Oxidant Effects of Rifampicin on Human Chronic Hepatitis C and Murine Acute Hepatocyte Disorder. Experimental and Therapeutic Medicine, 1, 1041-1047. https://doi.org/10.3892/etm.2010.159

[25] Saito, Z., Kaneko, Y., Kinoshita, A., Kurita, Y., Odashima, K., Horikiri, T., Yoshii, Y., Seki, A., Seki, Y., Takeda, H. and Kuwano, K. (2016) Effectiveness of Hepatoprotective Drugs for Anti-Tuberculosis Drug-Induced Hepatotoxicity: A Retrospective Analysis. BMC Infectious Diseases, 16, Article No. 668. https://doi.org/10.1186/s12879-016-2000-6

[26] Lang, S., Ortmann, J., Rostig, S. and Schiffl, H. (2019) Ursodeoxycholic Acid Attenuates Hepatotoxicity of Multidrug Treatment of Mycobacterial Infections: A Prospective Pilot Study. International Journal of Mycobacteriology, 8, 89-92. https://doi.org/10.4103/ijmy.ijmy_159_18

[27] Borzakova, S.N. and Reyzis, A.R. (2018) Principles of Diagnosis and Therapy of Drug-Induced Liver Injury in Children with Tuberculosis. Rossiyskiy Vestnik Perinatologii i Pediatrii, 63, 91-97. https://doi.org/10.21508/1027-4065-2018-63-3-91-97

[28] Chen, X., Xu, J., Zhang, C., Yu, T., Wang, H., Zhao, M., Duan, Z.H., Zhang, Y., Xu, J.M. and Xu, D.X. (2011) The Protective Effects of Ursodeoxycholic Acid on Isoniazid plus Rifampicin Induced Liver Injury in Mice. European Journal of Pharmacology, 659, 53-60. https://doi.org/10.1016/j.ejphar.2011.03.007 


\title{
Abbreviations Note List
}

\author{
ALT $=$ alanine transaminase \\ ALP $=$ alkaline phosphatase \\ AST $=$ Aspartate transaminase \\ ATD $=$ anti-tuberculosis drug \\ DILI $=$ drug-induced liver injury \\ FDA = U.S., Food and Drug Administration \\ $\mathrm{TB}=$ tuberculosis \\ $\mathrm{TID}=$ ter in die (three times a day) \\ UDCA = ursodeoxycholic acid \\ $\mathrm{ULN}=$ upper limit of normal
}

